\title{
Two Challenging Concepts in Mathematics Education: Subject- Specific Thoughts on the Complex Unit and Angles
}

\author{
Joaquin M. Veith ${ }^{1 *}$, Philipp Bitzenbauer ${ }^{2 *}$ \\ ${ }^{1}$ Institute for Mathematics and Applied Computer Science, Stiftung Universität Hildesheim, Hildesheim, GERMANY \\ ${ }^{2}$ Physics Education, Department of Physics, Friedrich-Alexander-Universität Erlangen-Nürnberg, Erlangen, GERMANY \\ * Corresponding author: veith@imai.uni-hildesheim.de \\ ** Corresponding author: philipp.bitzenbauer@fau.de
}

Received: 12 May $2021 \bullet$ Accepted: 15 Sep. 2021

Citation: Veith, J. M., \& Bitzenbauer, P. (2021). Two Challenging Concepts in Mathematics Education: Subject-Specific Thoughts on the Complex Unit and Angles. European Journal of Science and Mathematics Education, 9(4), $244-251$. https://doi.org/10.30935/scimath/11251

\begin{abstract}
:
In this paper, we focus on two particularly problematic concepts in teaching mathematics: the complex unit $\mathrm{i}$ and angles. These concepts are naturally linked via De Moivre's theorem but are independently misused in numerous contexts. We present definitions, notations, and ways of speaking about these terms from mathematics education that are not valid from a subject-specific point of view. We justify how these incorrectly used definitions, notations, and ways of speaking convey a false picture of the mathematical content and present options for introducing these concepts in classroom practice in a correct way.

Keywords: mathematics education, complex numbers, angles, misconceptions
\end{abstract}

\section{INTRODUCTION}

Mathematics is characterised by a very precise technical language. Thus, learning mathematics does not only require students to understand but also to use this highly specific and dense language (Wilkinson, 2015). If mathematical terms are not defined in a formally clean way, or if different definitions of a term coexist in mathematics education, this poses further hurdles in the use of this language for learners and may result in students' misconceptions (cf. Edwards \& Ward, 2008). This is not only of importance for teaching mathematics at all levels, but it is also particularly relevant for studies in mathematics education research, which examine students' conceptions of mathematical terms: Valid data on students' conceptions and understandings of mathematical concepts can only be collected if the mathematical concepts themselves are precisely defined and correctly used, at least by researchers and educators.

Two important concepts of any undergraduate mathematics course on Analysis or Linear Algebra are a) the complex unit $\boldsymbol{i}$ and b) angles. In this article, we demonstrate widespread definitions of, and ways of speaking about these terms that can be found in the literature on mathematics education, and we discuss subject-specific problems and serious errors that are related to these definitions (or rather approaches), and ways of speaking suggested in mathematics education literature. Thereby, we also provide empirical evidence for possible implications on student learning about the complex unit and angles. Finally, we suggest both, alternative ways of speaking as well as a mathematical rigorous definition of the complex unit $\boldsymbol{i}$ and angles, that could be suitable for classroom teaching. 


\section{The Complex Unit $\mathrm{i}$ in Mathematics Education}

Due to their historical significance and unusual naming (complex = complicated, imaginary = highly abstract), complex numbers are usually elevated to a special class of numbers (Nordlander \& Nordlander, 2011). However, they are a rather ordinary set extension of $\mathbb{R}$ which is even less complicated than the step from $\mathbb{Q}$ to $\mathbb{R}$. The essential starting point when teaching complex numbers is the introduction of the complex unit $i$. Various studies from mathematics education research of students' understanding of complex numbers revealed that the equation $i=\sqrt{-1}$ is an integral part of such introductory teaching sequences on complex numbers (Chin \& Jiew, 2020; Fehr, 1996; Scott, 2010). Sometimes this approach is even declared conducive to learning (Karakok et al., 2015; Sfard, 1991). Especially in didactic contexts focusing on complex numbers, however, we consider this approach unjustifiable, as it leads to fundamental misconceptions concerning complex numbers and real numbers. In the following, we point out the technical problems that are related to an introduction of the complex unit $i$ as the square root of -1 .

Even worse than the introduction of $i$ via the square root of -1 (which does not exist) is the definition of $i$ as $i:=\sqrt{-1}$ :

1. The symbol $\sqrt{ } \cdot$ is, up to this point, exclusively defined for non-negative real numbers. Thus $\sqrt{-1}$ is not a well-defined object and of similar informative value as the expression $\tan \frac{\pi}{2}$. Giving this expression a new name doesn't mean that one can do meaningful mathematics with it. And, as we will point out, a lot of work needs to be done before justifying such a notation.

2. Without any further elaboration of the new object $i=\sqrt{-1}$, one needs to assume that the used square root symbol is the same as the one used for real numbers and thus has the same properties. I.e. one merely extends the root operator to a new range of numbers. Of course, this does not work, and thus, leads to contradictions, the most striking one being:

$$
1=\sqrt{1}=\sqrt{(-1)^{2}}=\sqrt{-1} \cdot \sqrt{-1}=i \cdot i=-1
$$

In particular, then, justifications of the form

$$
\sqrt{-5}=\sqrt{-1} \cdot \sqrt{5}=i \sqrt{5}
$$

are inadmissible if they are done using the compatibility of the root function with real multiplication because this newly established root has quite different properties from the one defined for non-negative real numbers: for example, the identity

$$
\sqrt{a b}=\sqrt{a} \cdot \sqrt{b}
$$

becomes false defining $i:=\sqrt{-1}$ as shown in (1), the left- and the right-hand side will always differ by a root of unity. One can easily obtain more contradictions of this kind, and an empirical study on this specific topic suggests that the notion of $i$ being the square root of -1 can hinder students' understanding of complex numbers and the square root function in general (Chin \& Jiew, 2018).

3. Finally, the above definition does not define anything in a mathematical sense. The reason is that -1 has two square roots in $\mathbb{C}$, namely $i$ and $-i$, and it is not obvious from the above definition which one is meant. However, from an algebraic point of view, this does not matter: Both of the two solutions of the equation $x^{2}+1=0$ over $\mathbb{C}$, denoted by $i$ and $-i$ yield isomorphic fields (the so-called splitting field of $\left.f=x^{2}+1 \in \mathbb{R}[x]\right): \mathbb{R}(i) \cong \mathbb{R}(-i) \cong \mathbb{C}$. This is underlined by the fact that the complex conjugation $\tau: \mathbb{C} \rightarrow \mathbb{C}, a+i b \mapsto a-i b$ is an isomorphism.

Of course, it is possible to make mathematical sense of the equation $i=\sqrt{-1}$. However, this is anything but simple and requires a theory of complex analysis. In the end, it is clear: The equations $i=\sqrt{-1}$ and $-i=\sqrt{-1}$ are completely equal. While one can enforce the uniqueness of the root on the real numbers by their well-ordering, over $\mathbb{C}$ one arrives at uniqueness by specifying the branches of the holomorphic 
root function (Fritzsche, 2009). Hence, concerning introductory teaching approaches to complex numbers, it seems best to simply banish the commonly used equation $i=\sqrt{-1}$ for the above-mentioned reasons and instead write $i^{2}=-1$.

After all, how can complex numbers correctly be introduced in introductory courses? The answer can be found in numerous mathematical textbooks and is no secret: Usually, one constructs $\mathbb{C}$ from the real numbers via the quotient ring $\mathbb{R}[x] /\left(x^{2}+1\right)$ with the maximal ideal $\left(x^{2}+1\right)$. The quotient ring $\mathbb{R}[x] /\left(x^{2}+1\right)$ consists of the cosets $f+\left(x^{2}+1\right)$, where $f \in \mathbb{R}[x]$. However, this introduction requires an understanding of algebra that is too comprehensive for many areas of application of complex numbers. Fortunately, there is a rather simple (but correct) way to introduce the complex unit in introductory teaching approaches to complex numbers: Firstly, one defines two operations

$$
\begin{gathered}
\oplus: \mathbb{R}^{2} \times \mathbb{R}^{2} \rightarrow \mathbb{R}^{2} \\
((a, b),(c, d)) \mapsto(a+c, b+d)
\end{gathered}
$$

and

$$
\begin{gathered}
\odot: \mathbb{R}^{2} \times \mathbb{R}^{2} \rightarrow \mathbb{R}^{2} \\
((a, b),(c, d)) \mapsto(a c-b d, a d+b c)
\end{gathered}
$$

on $\mathbb{R}^{2}$. Secondly, one shows that the structure $\left(\mathbb{R}^{2}, \oplus, \odot\right)$ is a field. A real number $r$ is identified with the element $(r, 0)$ via the canonical embedding $\mathbb{R} \hookrightarrow \mathbb{R}^{2}$ and the element $(0,1)$ can be called $i$. Using the operations defined above, the following equation holds:

$$
i^{2}=(0,1) \odot(0,1)=(0 \cdot 0-1 \cdot 1,0 \cdot 1+1 \cdot 0)=(-1,0)=-1 .
$$

In summary, the great magic of the complex unit is dryly disenchanted using the presented approach:

\begin{abstract}
"Once we see that complex numbers are just pairs of real numbers, the previously mysterious status of the 'imaginary' number $\sqrt{-1}$ becomes much more prosaic. In fact, to the modern eye it is the 'real' numbers that are mysterious, because their rigorous definition involves analytic ideas such as sequences and convergence, which lead into deep philosophical waters and axiomatic set theory. In contrast, the step from $\mathbb{R}$ to $\mathbb{R}^{2}$ is essentially trivial [...]" (Stewart, 2014, p. 18).
\end{abstract}

Thus, there does not exist any necessity to write down the definition $i:=\sqrt{-1}$ in teaching an introductory course on complex numbers. Especially because it is not a definition.

On a last note, we state that the term imaginary unit often used for $i$ is most unfortunate for teaching and learning about complex numbers. As $(0,1) \in \mathbb{R}^{2}$ is an element of a real vector space, it can be visualised in the real plane as well or as badly as the numbers 0,1 or $\pi$ on the real number line. Thus, there is nothing imaginary about $i$ at all. Or, alternatively: Everything that mathematicians commonly call a number is an abstract construct of their minds rather than a real-world object. So technically speaking $i$ is real or imaginary to the same extent as all other numbers. One can choose a point of view, but there is no ontological difference between the numbers 1 and $i$.

The visualisation of complex numbers $a+i b$ in the real plane is simple using the absolute value $\sqrt{a^{2}+b^{2}}$ and the argument $\varphi=\arctan \frac{b}{a}$. Angles, therefore, play a crucial role here. There are many more conceptual inaccuracies about the concept of angles than about the complex unit $i$ which we will examine in the following section.

\title{
The Concept of Angles in Mathematics Education
}

Geometry links all the sub-fields of mathematics (Scheid \& Schwarz, 2007). Accordingly, the concept of angles plays a central role in mathematics and especially in mathematics education. However, the 
empirical investigation into students' misconceptions on angles reveals learners' difficulties on this topic (Biber et al., 2013; Ramdhani et. al., 2017; Sarkar et. al., 2020). Therefore, it is even more important to integrate the concept of angles into teaching in a non-contradictory way.

However, in many publications dealing with teaching angles, no mathematical definition for the term angle is provided at any point. Instead, it is only vaguely described and thus of no practical use at all: different notions are being mixed which then leads to confusing ways of speaking about this mathematical object (cf. Bütüner \& Filiz, 2016; Keiser, 2004; Koyunkaya, 2018; Richardson \& Koyunkaya, 2017). The mentioned papers have in common that they all state the difficulties and differences that come with the task of defining angles, and that those different approaches result in students' struggles with the angle concept (Keiser, 2000; White \& Mitchelmore, 1998). However, no ideas are being provided on how to lift this ambiguity, and the problems within the definitions themselves are not being addressed. Hence, in the following, we expatiate upon those problems and derive a proper definition. The informal definitions provided can be categorized into three key approaches which we will refer to as AP-1, AP-2 and AP-3 in the following (cf. Keiser, 2004; Richardson \& Koyunkaya, 2017):

AP-1: An amount of turning between two lines.

AP-2: The union of two rays with a common point.

AP-3: The interior region between the intersection of two lines.

The problems with AP-1 are described easily: If an angle is equated with an amount or magnitude, it can be treated like a physical quantity, meaning that one can add up or subtract angles, or divide them into smaller parts. Using those operations that are initially defined for numbers rather than geometrical objects will result in a conflict when mixed with AP-2. The key approach AP-2 introduces angles as geometrical figures but there exists no addition or subtraction on the set of figures in the plane, and there is nothing to gain from defining those since unions and intersections suffice. How mixing those concepts leads to very confusing ways of speaking can be studied well by looking at mathematics textbooks (cf. Figures 1-3). In Figure 1, the term calculating angles gets mentioned in the title and, further down, the text refers to measuring angles. Directly below, however, the angle $\beta$ itself is equated with $48^{\circ}$, i.e., a number with a unit, so an angle is shown as three-in-one simultaneously: a) a number with a unit, b) something one can draw and c) something one can measure.

\section{Calculate angles}

We can calculate angles greater than $180^{\circ}$ using the "full angle" $\left(360^{\circ}\right)$.

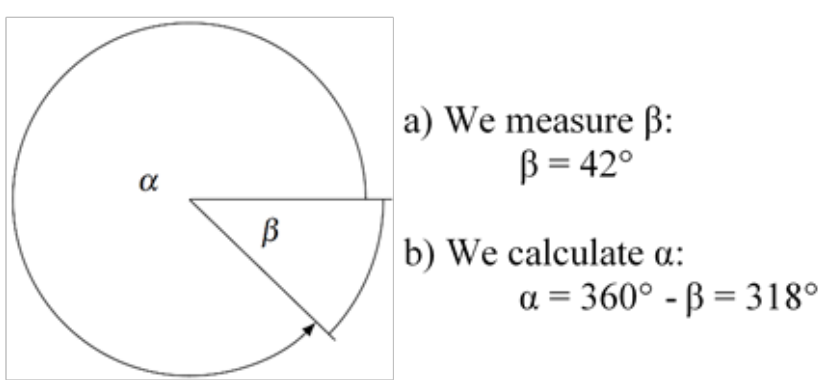

Figure 1. Typical representation of angles in classroom teaching adapted from a German mathematics school book (Becherer et al., 2006, p. 71)

This observation is supported by the following school book excerpt (cf. Figure 2): In this example, the discussion is about calculating and drawing angles. However, it is not clear what an angle is, i.e., what exactly is to be drawn. 


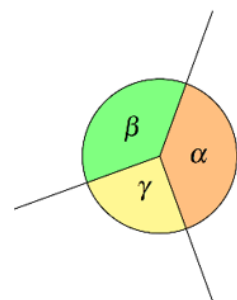

\begin{tabular}{|l|l|l|}
\hline$\alpha$ & $\beta$ & $\gamma$ \\
\hline $140^{\circ}$ & $95^{\circ}$ & \\
\hline & $210^{\circ}$ & $45.5^{\circ}$ \\
\hline $87^{\circ}$ & $184^{\circ}$ & \\
\hline
\end{tabular}

Figure 2. Figure from a typical school book exercise on angles. The corresponding task is the following: "The three angles $\alpha, \beta$ and $\gamma$ together make a full circle angle. Draw the given angles in the table and measure the missing angle in your drawing. Check your drawing by calculating the missing angle" (Esper et al., 2007, p. 44)

In numerous textbooks, all the concepts about angles are mixed up as well. For example, in the task presented in Figure 3, one is supposed to draw angles, add them up, measure them and estimate their size.
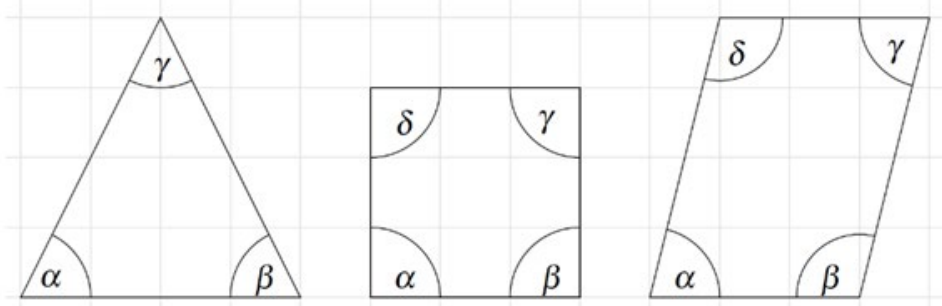

Figure 3. Figure from a typical school book exercise on angles. The corresponding task is the following: "Draw the three figures with quadruple side lengths. Measure the angles and sum them up. What do you notice?" (Leppig et al., 2006, p. 71)

It is often not clear from such school book texts and exercises what an angle is. Statements of the form "The angle $\alpha=180^{\circ}$ " or "The sum of all inscribed angles in a triangle is $180^{\circ}$ " may serve as examples. Quite absurd, but in a sense, comparable statements would be "The triangle $4 \mathrm{~cm}^{2 \prime}$ or "The sum of cube A and cube B is 4 litres". With angles, however, such statements are not met with astonishment. This is based on a lack of differentiation between geometric objects and their measures (cf. Table 1).

Table 1. Geometric objects, their measures and corresponding units

\begin{tabular}{lcc}
\hline Geometric object & Measure & Unit \\
\hline Stretch & Length & $1 \mathrm{~m}$ \\
Figure & Area & $1 \mathrm{~m}^{2}$ \\
Body & Volume & $1 \mathrm{~m}^{3}$ \\
Angle & Angle measure & $1^{\circ}$ \\
\hline
\end{tabular}

Of course, like any other geometric object, an angle cannot be equated with its measure, and one has to define the object itself first (so in a sense, AP-1 seems to be the worst approach). Angles are often visualised using their legs and a small circular arc (cf. Figure 4). But which of these is the angle?

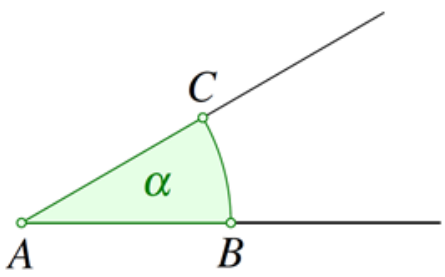

Figure 4. Representation of angles

Suppose one defines the green figure as an angle. In that case, the term circle sector is no longer needed and furthermore, it seems absurd to introduce a new measurement for something that is a figure from 
a mathematical point of view. The same argument also applies to the area of the plane enclosed by the two rays (especially since it must then be clarified which of the two areas it should be). So, AP-3 also does not capture the essence of an angle. Furthermore, equipping the set of angles with a measure will conflict with this approach, as we will elaborate below. If one only takes the boundary of the green figure or a part of it, one deals with line segments and circular lines, i.e., objects for which there is also already a measure, namely the length. Consequently, none of the approaches AP-1, AP-2 and AP-3 satisfies an introduction of angles that would not immediately lead to problems or contradictions. So how do we define an angle? In any case, three points can unambiguously define an angle and to each angle we can associate at least three points.

For introducing angles in mathematics teaching, we propose the following definition of the term angle to properly distinguish between the geometric object angle and its measure, namely the angle measure (cf. Table 1).

Definition: Given three points A, B and C of the Euclidean plane. Then the ordered tuple

$$
([A B,[A C)
$$

is called angle between $[A B$ and $[A C$. The rays $[A B$ and $[A C$ are called sides (or legs) and $A$ the vertex of the angle.

Hence, an angle cannot be visualised directly; in particular, an angle is not a figure. Instead, an angle is visually represented by two half-lines. Similarly, the mapping rule of a function is visually represented by a graph.

With this definition, it is clear that we are dealing with a new geometric object for which one must introduce a new measure. Before this is possible, however, the set of angles must be compatible with a measure from a mathematical point of view. This means, that one first has to equip this set of angles with the structure of a $\sigma$-algebra and then define a measure on this algebra. Not before then it is possible to have a way of speaking and notation compatible with the rest of geometry: a line segment $[\mathrm{AB}]$ of length $\overline{\mathrm{AB}}=\mathrm{d}(\mathrm{A}, \mathrm{B})$, a figure $\mathrm{F}$ with area $\mathrm{A}(\mathrm{F})$, a solid $S$ with volume vol(S) and finally an angle $\alpha:=$ ( $[A B,[A C)$ with angular measure $\mu(\alpha)$. In this context, a relation on the set of angles can also be introduced via the concept of measurement in order to provide statements such as "angle $\alpha$ is greater than angle $\beta$ " with meaningfulness. However, the phrase calculating an angle cannot be saved because what is meant is always measurement as for any other geometric object. It is precisely these fine distinctions that are necessary in order not to confuse all mathematical operations in the context of angles.

A more messy but convenient alternative is possible by defining angles as circular arcs with radius 1 . Then one can at least draw an angle because one has not defined anything new. In this way, a measure can also be obtained for free - one simply takes the curve integral between the start and end point of the angle and subtracts $2 \pi$ from the value of the integral until it lies in the interval $[0,2 \pi]$ - also known as radian measure.

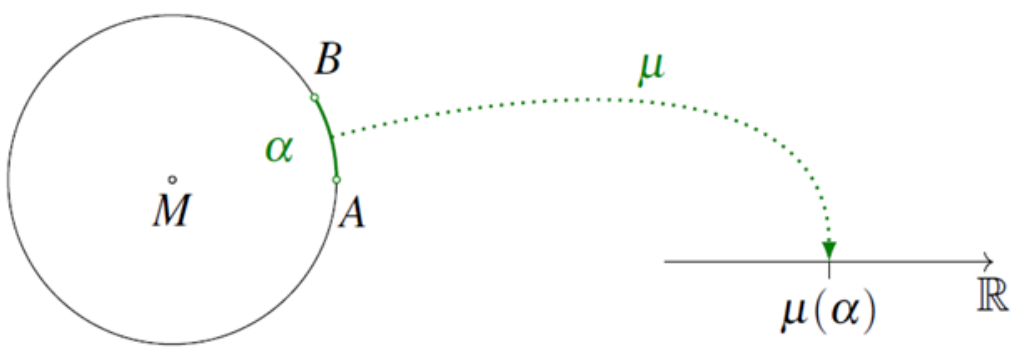

Figure 5. Visualisation of the radian measure's meaning 
However, the latter term could be deleted without replacement and instead simply say length since one has only measured the length of a curve. Moreover, the question of why the term angle is introduced at all remains unanswered. Alternatively, one could answer honestly: It is complicated.

When it comes to teaching the concept of angles at school, there is probably no other option than to establish the degree measure and its arbitrariness (a full angle measures $360^{\circ}$ because 360 has many divisors) without any justification. However, in the course of didactic elementarisation, one must not elementarise away everything that distinguishes it as a mathematical object. Otherwise, an illogical and impenetrable mixture of different concepts and terms emerges.

Lastly, we summarize our arguments above into three central misconceptions on angles that we have extracted from literature on mathematics education:

1. Equating angles with their measure (Keiser, 2004; Biber et al., 2013).

2. Using operations for angles that are (a priori) not defined for them, i.e. summing/subtracting, or arrange them in a certain order (Biber et al., 2013; Richardson \& Koyunkaya, 2017).

3. Speaking of or referring to "drawing" angles but not defining them in a way where this phrase is justified (Bütüner \& Filiz, 2016; Richardson \& Koyunkaya, 2017; Sarkar, Kadam \& Pillai, 2020).

It is important to note that these misconceptions are not independent from one another. If one does not differentiate between an angle and its measure, it is more likely that he or she treats the angle like the number associated with its measure, so conception 2 is somewhat implied by conception 1 . As for conception 3, drawing angles requires a specific definition where an angle is a defined as a figure, which for the above-mentioned reasons is not a good idea in general, even though it is easily possible by using circular arcs. The important part here is that this way of speaking leads to immanent problems when working with multiple different definitions at once, as we clarified above. We assigned publications to this conception if the phrase "drawing" was mentioned since none provided any mathematical definition of an angle. Furthermore, the informal descriptions of angles were either

- strictly false, e.g. "An angle is an intersection of two rays at the same end point" (Bütüner \& Filiz, 2016) or

- of no practical use, e.g. "An angle is the interior region between the intersection of two lines" (Richardson \& Koyunkaya, 2017).

\section{CONCLUSION}

As explained in this article using the examples of the complex unit $i$ and the angle, some mathematics objects that seem elementary at first glance are based on an extensive theory that only reveals itself at a second glance. If one ignores this second look, one runs the risk of simplifying these concepts to such an extent that technical errors occur, which manifest themselves in incorrect ways of speaking, notations and generally incorrect use of mathematical content. In particular, this contradicts the general claim of mathematics to establish a clear and consistent technical language. Although one is often forced to suppress certain technical aspects in classroom practice within the framework of didactic reconstruction, this should always be done in an upwardly compatible manner. After all, how are learners supposed to develop a correct technical language and a well-founded idea of mathematical content if already the basics are taught incorrectly?

Author contributions: All authors were involved in concept, design, collection of data, interpretation, writing, and critically revising the article. All authors approve final version of the article.

Funding: The authors received no financial support for the research and/or authorship of this article.

Declaration of interest: Authors declare no competing interest.

Data availability: Data generated or analysed during this study are available from the authors on request. 


\section{REFERENCES}

Biber, C., Tuna, A., \& Kormaz, S. (2013). The mistakes and the misconceptions of the eighth grade students on the subject of angles. European Journal of Science and Mathematics Education, 1(2), 50-59. https://doi.org/10.30935/scimath/9387

Bütüner, S. Ö., \& Filiz, M. (2016). Exploring high-achieving sixth-grade students' erroneous answers and misconceptions on the angle concept. International Journal of Mathematical Education in Science and Technology, 48(4), 533-554. https://doi.org/10.1080/0020739X.2016.1256444

Chin, K. E., \& Jiew, F. F. (2018). A framework of making sense of mathematics. Proceedings of the $8^{\text {th }}$ ICMI-East Asia Regional Conference on Mathematics Education: EARCOME 8, 309-319.

Chin, K. E., \& Jiew, F. F. (2020). Knowing and Grasping of Two University Students: The Case of Complex Numbers. The Mathematics Enthusiast, 17(1), 273-306.

Edwards, B., \& Ward, M. B. (2008). The Role of Mathematical Definitions in Mathematics and in Undergraduate Mathematics Courses. In M. P. Charlson \& C. Rasmussen (Eds.), Making the connection: research and teaching in undergraduate mathematics education (MAA notes, p. 223-232). Mathematical Association of America. https://doi.org/10.5948/UPO9780883859759.018

Esper, N., Lütticken, R., \& Schornstein, J. (2007). Fokus Mathematik Gymnasium 6 [Focus on mathematics high school 6]. Corneslen Verlag.

Fehr, R. (1996). More emphasis on Complex Numbers? Twenty-Ninth Annual Meeting - Florida Section Mathematical Association of America. https://ralphfehr.com/files/maapaper.pdf

Fritzsche, K. (2009). Grundkurs Funktionentheorie [Basic course in function theory]. Spektrum Akademischer Verlag.

Karakok, G., Soto-Johnson, H., \& Dyben, S. A. (2015). Secondary teachers' conception of various forms of complex numbers. Journal of Mathematics Teacher Education, 18(4), 327-351. https://doi.org/10.1007/s10857-014-9288-1

Keiser, J. M. (2000). The role of definition. Mathematics Teaching in the Middle School, 5(8), 506-511. https://doi.org/10.5951/MTMS.5.8.0506

Keiser, J. M. (2004). Struggles with developing the concept of angle: Comparing sixth-grade students' discourse to the history of the angle concept. Mathematical Thinking and Learning, 6(3), 285-206. https://doi.org/10.1207/s15327833mt10603_2

Koyunkaya, M. Y. (2018). An examination of a pre-service mathematics teacher's mental constructions of relationships in a right triangle. International Journal of Education in Mathematics, Science and Technology, 6(1), 58-78. https://doi.org/10.18404/ijemst.328344

Leppig, M., et al. (2006). Lernstufen Mathematik 6 [Mathematics levels 6]. Cornelsen Verlag.

Mitchelmore, M., \& White, P. (2000). Development of angle concepts by progressive abstraction and generalisation. Educational Studies in Mathematics, 41(3), 209-238. https://doi.org/10.1023/A:1003927811079

Nordlander, M., \& Norlander, E. (2011). On the concept imiage of complex numbers. International Journal of Mathematical Education in Science and Technology, 43(5), 627-641.

Ramdhani, M. R., Usodo, B., \& Subanti, S. (2017). Discovery learning with scientific approach on geometry. Journal of Physics: Conference Series, 895(1), 012033. https://doi.org/10.1088/1742-6596/895/1/012033

Richardson, S. E., \& Koyunkaya, M. Y. (2017). Fostering students' development of the concept of angles using technology. Australian Primary Mathematics Classroom, 22, 13-20.

Sarkar, P., Kadam, K., \& Pillai, J. S. (2020). Learners' approaches, motivation and patterns of problem-solving on lines and angles in geometry using augmented reality. Smart Learning Environments, 7, 17. https://doi.org/10.1186/s40561-020-00124-9

Scott, P. (2010). I am needed. The Australian Mathematics Teacher, 66(1), 3-4.

Sfard, A. (1991). On the dual nature of mathematical conceptions: Reflections on processes and objects as different sides on the same coin. Educational Studies, 22(1), 1-36. https://doi.org/10.1007/BF00302715

Stewart, I. (2014). Galois theory (4th ed). CRC Press https://doi.org/10.1201/b18187

Wilkinson, L. C. (2015). The language of learning mathematics. The Journal of Mathematical Behavior, 40, 2-5. https://doi.org/10.1016/j.jmathb.2015.04.001 